\title{
Physical parameters in extragalactic star forming regions
}

\author{
R. MAUERSBERGER, C. HENKEL, AND L.J. SAGE \\ MPIfR, Auf dem Hügel 69, D-5300 Bonn, F.R.G.
}

To use a molecule as a diagnostic of a single star forming region,.one has to observe it in different transitions. That is especially true for galaxies, where the beam filling factor is small and unknown. From studies of Galactic objects, the CS molecule is a good tracer of dense gas. Toward the IR galaxies NGC 253, IC 342 and M 82, Mauersberger and Henkel (1989) observed the $J=2-1,3-2$ and 5-4 transitions of CS at 3,2 and $1.3 \mathrm{~mm}$ wavelength, repectively. They also measured emission of the isotope $\mathrm{C}^{34} \mathrm{~S}$, which has an abundance $\sim 1 / 23$ that of the main isotope. This study revealed that the $\mathrm{H}_{2}$ densities in the circumnuclear gas exceed $10^{4}$, and, in the case of NGC $253,10^{5} \mathrm{~cm}^{-3}$. In all three cases, the distribution of the dense gas traced out by CS follows the CO distribution.

In order to confirm this high density for NGC 253, we (Mauersberger et al., 1990) conducted a multi-level study of $\mathrm{HC}_{3} \mathrm{~N}$ (cyanoacetylene) toward NGC 253. We measured six transitions of this heaviest molecule detected outside our Galaxy. Comparing the relative intensities of the $J$ components (Fig. 1) we reached the following conclusions:

1. The $3 \mathrm{~mm}$ transitions can only be fitted if the bulk of the gas seen in $\mathrm{HC}_{3} \mathrm{~N}$ has a density of $\sim 10^{4} \mathrm{~cm}^{-3}$ and a temperature of $\sim 30 \mathrm{~K}$. The filling factor should be comparable to $\mathrm{CO}$ (i.e. $>10 \%)$.

2. This component cannot reproduce the observed emission of the $2 \mathrm{~mm}$ and $1 \mathrm{~mm}$ lines of $\mathrm{HC}_{3} \mathrm{~N}$. These lines require a gas component with a much higher excitation. The high excitation model applied to fit the high $J$ tail is a conservative guess and has as parameters $n\left(\mathrm{H}_{2}\right)=10^{5} \mathrm{~cm}^{-3}$ and $T_{\text {kin }}=150 \mathrm{~K}$. The filling factor should be low $(0.1 \%)$.

3. The existence of two components is very similar to our own Galactic center, where Walmsley et al. (1986) have detected two components, the denser one being less excited than the dense component toward NGC 253.

Besides the studies summarized above, there are a number of other methods of determining the state of the densest molecular gas in external galaxies:

$\mathrm{H}_{2} \mathrm{CO}$. In our Galaxy, the $2 \mathrm{~cm}$ line of formaldehyde is normally seen in absorption. Only toward a handfull of Galactic sources, thermal emission has been observed. The explanation of the emission requires densities exceeding $10^{5} \mathrm{~cm}^{-3}$. 2-cm emission toward M 82 was detected by Baan et al. (1990).

$\mathrm{N}_{2} \mathrm{H}^{+}$. This molecular ion, the third detected outside the Galaxy, has been measured toward five galaxies and has been mapped toward NGC 253 and $\mathrm{M} 82$. Unlike other molecules, $\mathrm{N}^{2} \mathrm{H}^{+}$seems to be centrally peaked toward $\mathrm{M} 82$.

$\mathrm{CH}_{3} \mathrm{CCH}$. Methyl acetylene is the most complex molecule detected outside our galaxy, namely toward M82. It is, however, difficult to disentangle its K-lines from the velocity structure of Galaxies. 


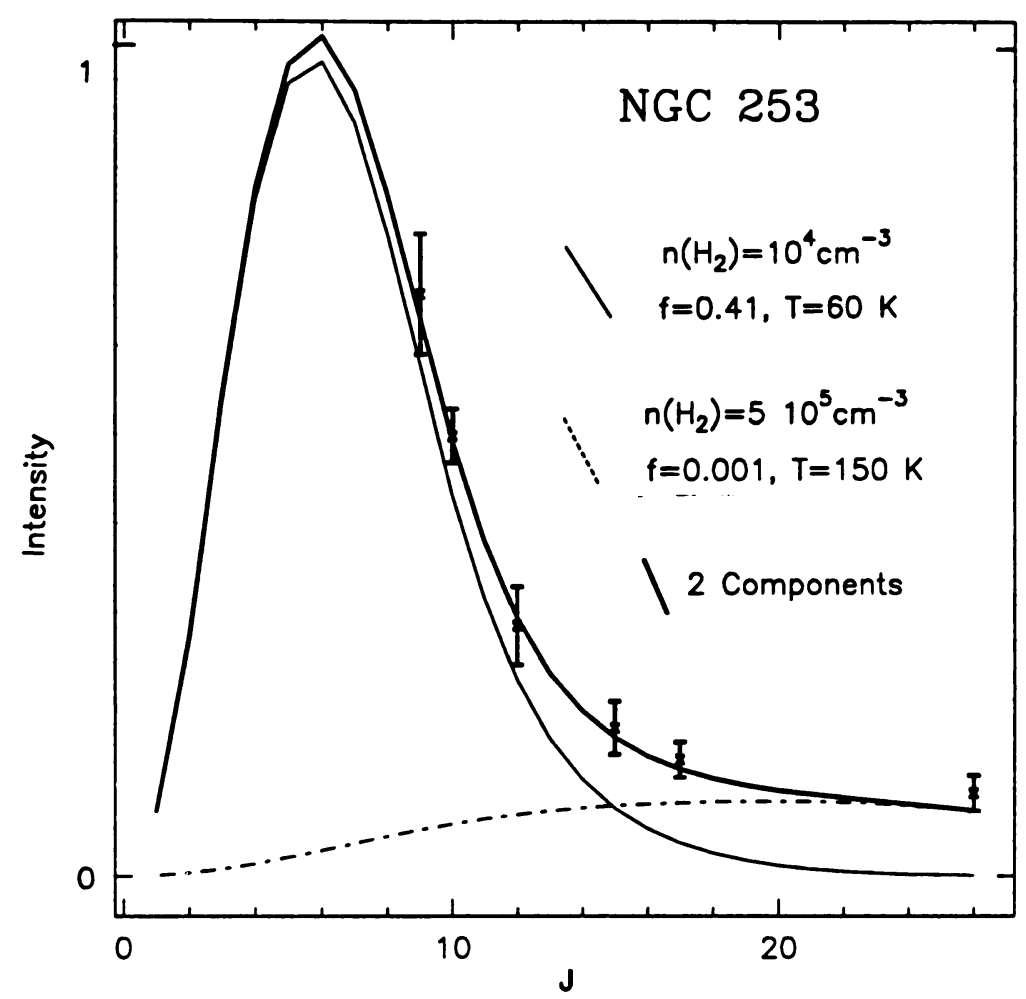

Fig!1: Fits to our data with a two component model. Intensjties are scaled with respect to the maximum emission. The thick line represents a two component model fitting the measured data. The thin and the dashed lines show the contribution of each of the two components. The parameter for the relative $\mathrm{HC}_{3} \mathrm{~N}$ abundance per velocity gradient, $X(\mathrm{CO}) /\left(d_{t} / d r\right)$, has been chosen to be $510^{-10} /\left(\mathrm{km} \mathrm{s}^{-1} / \mathrm{pc}\right)$.

Unlike in the center of our Galaxy, $\mathrm{CH}_{3} \mathrm{CCH}$ emission is stronger than that of $\mathrm{CH}_{3} \mathrm{CN}$.

$\mathrm{SiO}$. $\mathrm{SiO}$ is mainly observed toward regions with a very elevated temperature and in shocked gas. It could therefore be used as a 'chemical thermometer'. We have detected its $J=2-1$ line of the vibrational ground state $(v=0)$ toward the nucleus of NGC 253 but not toward M 82 and IC 342. It may be that temperatures in NGC 253 are higher than in the other two galaxies.

\section{References}

Baan, W., Henkel, C., Schilke, P., Mauersberger, R., Güsten, R.: 1990, Astrophys. J. 353, 132 Henkel, C., Mauersberger, R., Schilke, P.: 1988, Astron. Astrophys.201, L23

Mauersberger, R., Henkel, C.: 1989, Astron. Astrophys. 223, 79.

Mauersberger, R., Henkel, C., Sage, L.J.: 1990, Astron. Astrophys., in press

Walmsley, C.M., Güsten, R., Angerhofer, P., Churchwell, E.: 1986, Astron. Astrophys. 155, 129 